\title{
On the maximal noise for stochastic and QCD traveling waves
}

\author{
Robi Peschanski* \\ Institut de Physique Théorique \\ Unité de Recherche associée au CNRS \\ CEA-Saclay, F-91191 Gif/Yvette Cedex, France.
}

\begin{abstract}
Using the relation of a set of nonlinear Langevin equations with reaction-diffusion processes, we note the existence of a maximal strength of the noise for the stochastic traveling wave solutions of these equations. Its determination is obtained using the field-theoretical analysis of branching-annihilation random walks near the directed percolation transition. We study its consequence for the stochastic FisherKolmogorov-Petrovsky-Piscounov equation. For the related Langevin equation modeling the Quantum Chromodynamic nonlinear evolution of the gluon density with rapidity, the physical maximal-noise limit may appear before the directed percolation transition, due to a shift in the traveling-wave speed. In this regime, an exact solution is known from a coalescence process. Universality and other open problems and applications are discussed in the outlook.
\end{abstract}

\section{Introduction}

The aim of the present note is to discuss some aspects of traveling wave solutions of a class of nonlinear equations with noise. These nonlinear Langevin equations are all related to the continuum limit of reaction-diffusion processes in statistical physics.

*e-mail: robi.peschanski@cea.fr 
A typical exemple of this class of equations is the stochastic FisherKolmogorov-Petrovsky-Piscounov (sFKPP) equation. This equation reads

$$
\frac{\partial w(x, t)}{\partial t}=D \frac{\partial^{2} w}{\partial x^{2}}+\lambda\left(w-w^{2}\right)+\epsilon \sqrt{w(1-w)} \nu(x, t)
$$

where the white noise verifies $\langle\nu\rangle=0$ and

$$
\left\langle\nu(x, t) \nu\left(x^{\prime}, t^{\prime}\right)\right\rangle=\delta\left(x-x^{\prime}\right) \delta\left(t-t^{\prime}\right) .
$$

The deterministic part of (1) is the well-known FKPP equation [1]. $D$ is the diffusion coefficient, $\lambda$ characterizes the activation strength since it is responsible for an exponential grow of the FKPP solution in the dilute regime (when $w^{2} \ll w$ ). At some point the nonlinear damping term becomes effective when entering the dense regime. The fluctuation contribution which sensibly modifies the solutions as we shall discuss in length, is characterized by the value of $\epsilon$, the noise strength.

The same type of equation appears in various fields of statistical physics and, recently, in the domain of Quantum Chromodynamics (QCD), the interaction theory of quarks and gluons, where it happens to model [2] the evolution of the gluon momenta in the wave-function of a hadron or a nucleus when the energy increases.

The main property of the FKPP equation [3] is to admit "universal" traveling wave solutions. Indeed, one finds in a quite general way travelingwave solutions of the form $w(x-v t)$ where the speed $v$ is independent of the initial conditions (provided they are sharp enough in $x$ ). The reason is that a critical speed is selected after some evolution in time. Qualitatively, this property may be considered as a critical non-equilibrium mechanism since it corresponds to a solution flowing from an unstable $(w=0)$ to a stable $(w=1)$ fixed point. It results from the "strain" between the exponential increase raised up by the initial conditions in the linear regime and the damping due to the nonlinear terms. Subasymptotic terms of the speed and the structure of the wave front in the so-called "leading edge" region [4] can also be predicted. There are many results and applications of this traveling wave property in the literature [4].

In physical problems, one has often to take into account the existence of fluctuations. One simple exemple is the application of an evolution equation for a discrete set of "walkers" on a lattice generating statistical fluctuations at the edge, whose first approximation in the continuum limit is a cut-off, 
e.g. on the function $w$ of Eq.(1) for the sFKPP equation. Indeed, it has been shown that, even with only a tiny cut-off [5], the effect on the asymptotic solutions is sizeable. The effect of fluctuations in the dilute part of the system (near $w=0$ ) has been confirmed by simulations [6]. It is amplified for two reasons. If of negative sign, a random fluctuation can drive the system to $w=0$ where it stays indefinitely, and thus acting as an effective cut-off. If positive, the fluctuation is amplified by the exponential increase in the dilute region and extends its effect far into the dense region. Both effects have been analyzed and clearly identified in the weak-noise regime [7]. In fact, the solution may be interpreted as a stochastic combination of traveling waves with some dispersion $\Delta$ and the average solution changes from a function of $x-v t$ to a function of $\frac{x-v t}{\Delta \sqrt{t}}$. In particle physics it corresponds to a transition from "geometrical scaling" [8] to "diffusive scaling" [9] properties of the gluon momentum distribution.

In the strong-noise regime, the fluctuations are expected to have a decisive effect in low-dimensional problems. Indeed in the case of the sFKPP equation, an exact solution can be found using a duality relation with reversible reaction processes at the coalescence limit [10, 11]. It has also been applied to the QCD case [12]. In both cases, the exact solution features noisy traveling waves and correlations fully dominated by the strongest fluctuations.

However, one may question whether one can go to arbitrary strength of the noise. Indeed, as we shall see through the general relation of the Langevin equations with reaction-diffusion processes, one has to take into account the existence of a phase transition which belongs to the universality class of the directed percolation (dp). At some point, the system no more propagates.

Hence we are led for our purpose to take into account the fluctuations in an appropriate framework. The study of fluctuations is where the fieldtheoretical formalism is certainly useful, since they are properly taken into account in the very definition of the action. Our aim is thus to investigate what new information the field-theoretical formalism of reaction-diffusion processes can bring to the traveling-wave problem for the class of Langevin equations to which sFKPP and the QCD evolution equations belong. The field-theoretical formalism for reaction-diffusion processes [13, 14, 15] has been already used in the context of the weak-noise regime of traveling waves [16], where it confirmed the results of the cut-off analysis of Ref. [5]. Our aim in the present note is to use the field-theoretical formalism in the full range of noise strength, and more precisely at the strong-noise limit, where 
renormalization effects allow for a proper account of the large fluctuation effects.

In fact the main tool used of our note is to take benefit of a thorough fieldtheoretical analysis [14] of specific reaction diffusion process, the Branching Annihilation Random Walks (BARW), which we will relate to the strong noise problem of the class of Langevin equation we want to investigate. Indeed, the continuum limit of BARW processes leads to a Langevin equation of the same class. Hence the renormalization analysis of the BARW process leads to the existence of a maximal noise strength and to its evaluation. We will argue that this result extends to the class of langevin equations we study. However, the specific case of QCD will need a supplementary analysis, due to a different term in the corresponding Langevin equation.

The plan of our note is the following: In section 2 , we briefly recall some aspects of the Langevin equations applied to reaction-diffusion and QCD processes and in section 3, we infer the existence of the maximum noise strength from the study of the BARW process and give an evaluation. In section 4, we apply our result to the two exemples of the sFKPP equation and to the QCD nonlinear evolution. We close this note in section 5 by a summary of our results, with a discussion on the universality range of our result (for more general reaction-diffusion) and on possible applications to QCD.

\section{Langevin equations and reaction-diffusion processes}

\section{Field theoretical formalism of the Langevin equations}

It is known that Langevin equations of sFKPP type can be analyzed in the form of a bosonic quantum field theory [13]. This formalism is particularly convenient to treat fluctuations superimposed to mean-field equations of FKPP type, since they are intrinsically taken into account by specific terms in the field-theoretical action. For instance, the field theory corresponding to Eq.(1) is defined in terms of bosonic fields $w$ and their auxiliary counterparts $\bar{w}$ by the action

$$
S[w, \bar{w}]=\int d^{d} x d t\left\{\bar{w}\left[\partial_{t}+D \nabla^{2}\right] w-\lambda \bar{w} w(1-w)-\frac{\epsilon^{2}}{2} \bar{w}^{2} w(1-w)\right\} .
$$


In fact these auxiliary fields appear as external source fields for the deterministic (for linear terms in $\bar{w}$ ) and the noise terms (for quadratic terms in $\bar{w})$. Let us illustrate the field-theoretical formulation [13] with the example of (3): performing the path integral over the terms linear in the auxiliary field $\bar{w}$ gives rise exactly to the mean-field terms which, in the case of (1) is nothing else than the FKPP equation. Performing the gaussian path integral of the quadratic part in $\bar{w}^{2}$ allows one to include the fluctuation contribution and generates correspondingly the noise term in the sFKPP version (1) of the Langevin formalism.

This field-theoretical framework casts a useful bridge between the methods of quantum field theory and the solution of reaction diffusion processes and with the treatment of equations of the sFKPP type (Ref.[14] will be of particular interest for us, while for a comprehensive review, see [15]). Indeed, in such reaction-diffusion processes it is possible to discuss the probability of evolution from an "inactive" state to an "active" one, under the effect of branching (or splitting), diffusion, annihilation and merging (or coagulation) of random walkers. In the continuum limit, it contains in full generality the field-theoretical description of the Langevin equations of the type (1). We put this connection on a precise footing in the following sections.

One major interest of the field-theoretical treatment of the reactiondiffusion system is the treatment of fluctuations which play an important role in low-dimensional systems, such in our $d=1$ case. It appears in particular through the renormalization of the field theory. In fact due to fluctuations, a phase transition happens which is in the universality class of directed percolation [14, 15].

\section{Langevin Equation for QCD evolution processes}

In the QCD problem the region near the "inactive" state corresponds to the boundary of the dilute region for the gluon density (or "transparency" where the S-matrix $S=1$ ) while the "active" state corresponds to the boundary of the dense region (or "strong absorption" where the S-matrix $S=0$ ), see Fig.1 and Ref.[17] for a review.

The QCD evolution process which we refer to is thus the rapidity dependence of the tranverse-momentum gluon distribution in a nucleon or nucleus

\footnotetext{
${ }^{1}$ It is even more general since some reaction-diffusion processes cannot be described by a consistent Langevin equation (e.g. generating an imaginary noise term).
} 


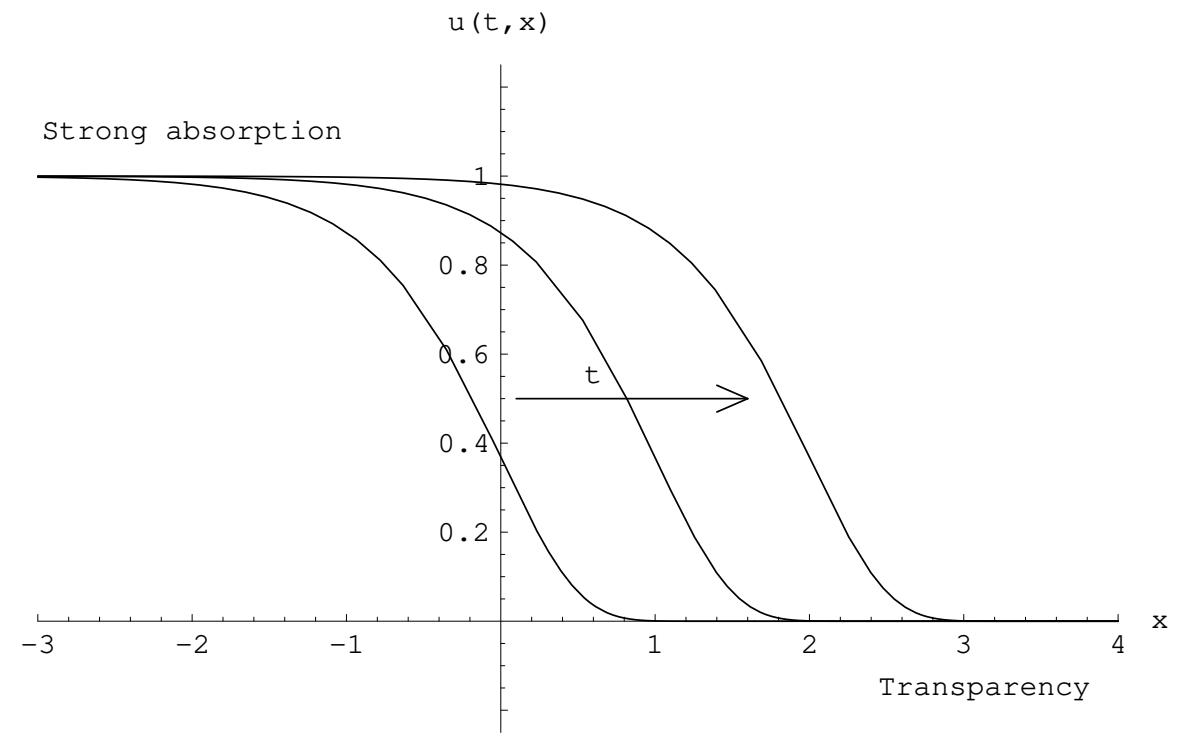

Figure 1: QCD traveling waves, from Ref.[21].

The traveling waves are mean field solutions of QCD evolution equations, translationally invariant in time and joining the unstable fixed point(transparency or dilute medium of gluons), to the stable fixed point (strong absorption or dense gluon medium).

target [17] which evolves from the transparency to the strong absorption regime when the rapidity (i.e. the logarithm of the energy) increases. This equation, at least within an approximate framework can be given a fieldtheoretical interpretation in terms of non-equilibrium processes.

Indeed, a coarse-graining approximation [18] leads to a simplified description based on the stochastic equation

$$
\partial_{Y} T(L, Y)=\chi\left(-\partial_{L}\right) T(L, Y)-T^{2}(L)+\sqrt{\kappa \alpha_{s}^{2} T(L, Y)} \nu(L, Y) .
$$

where $L=\log k^{2}, k$ is the gluon transverse momentum, $Y$ the rapidity in units of the strong coupling constant $\alpha_{s}$ and $x$ the fraction of total energy brought by the gluon. We will consider $\chi$ as the leading order in $\alpha_{s}$ BalitskyFadin-Kuraev-Lipatov (BFKL) kernel [19]

$$
\chi(\gamma)=2 \psi(1)-\psi(\gamma)-\psi(1-\gamma) .
$$


Modifications by higher order corrections will not be introduced at the present stage of our derivation but they certainly occur and deserve further study. The noise $\nu(L, Y)$ satisfies $\langle\nu\rangle=0$ and

$$
\left\langle\nu(L, Y) \nu\left(L^{\prime}, Y^{\prime}\right)\right\rangle=\frac{2}{\pi} \delta\left(Y-Y^{\prime}\right) \delta\left(L-L^{\prime}\right) .
$$

The deterministic part of (41) is the Balitsky-Kovchegov (BK) equation [20]. Its solutions have been found to converge to traveling waves after some rapidity evolution [21]. This fact is particularly clear if one uses the diffusive approximation as we shall see now. It leads to a deterministic part of the equation identical to FKPP with a modified noise term.

Indeed, expanding the BFKL kernel to second order around some $\gamma_{c}$,

$$
\chi(\gamma)=\chi_{c}+\chi_{c}^{\prime}\left(\gamma-\gamma_{c}\right)+\frac{1}{2} \chi_{c}^{\prime \prime}\left(\gamma-\gamma_{c}\right)^{2}=A_{0}+A_{1} \gamma+A_{2} \gamma^{2}
$$

The value of $\gamma_{c}$ is obtained through the equation $\gamma_{c} \chi^{\prime}\left(\gamma_{c}\right)=\chi\left(\gamma_{c}\right)$ which determines the critical speed of the traveling waves. In fact, it can be shown [21] that $\gamma_{c}$ plays the role of an anomalous dimension characterizing the wave front formed by the "blocking" mechanism described in introduction.

Introducing the space $(x)$ and time $(t)$ variables by the redefinition [21]

$$
t=\alpha_{s} Y, \quad x=L-A_{1} \alpha_{s} Y, \quad u(x, t)=\frac{T}{A_{0}},
$$

Eq.(4) gets mapped, within the diffusive approximation (7), onto the Langevin equation:

$$
\frac{\partial u(x, t)}{\partial t}=A_{2} \frac{\partial^{2} u}{\partial x^{2}}+A_{0}\left(u(x, t)-u^{2}(x, t)\right)+\frac{2 \alpha_{s}}{\pi} \sqrt{\kappa A_{0} u(x, t)} \nu(x, t) .
$$

It is thus easy to realize that the first term $A_{0}$ corresponds to branching, $A_{2}$ to the diffusion constant. The space derivative term with coefficient $A_{1}$ in (7), is thus translated into a time-dependent shift on the space variable by the redefinition (8) of the kinematic variables. This shift plays, as we shall see, an important role in the application of reaction-diffusion processes to QCD and to the maximal noise problem in particular.

Hence, in the coarse-graining approximation of QCD evolution process, the deterministic part of (9) can be interpreted as gluon momentum diffusion, branching and merging while the noise $\nu$ is a simplified description of 
the gluon-number fluctuation effects, which in the diagrammatic QCD framework is associated to the so-called "Pomeron loops". The strength of these fluctuations is proportional to $\alpha_{s}$ while the coarse-grained fudge factor $\kappa$ may be large.

Writing

$$
D \equiv A_{2}, \quad \lambda \equiv A_{0}, \quad \epsilon \equiv \frac{2 \alpha_{s}}{\pi} \sqrt{\kappa A_{0}},
$$

Eq.(9) is similar to the sFKPP equation (11), except for the quadratic term in the noise which is here absent.

In the Langevin formulation, the quadratic term $-w^{2}$ in the noise of (11) cancels the fluctuations near the stable fixed point of the mean-field equation at $w=1$, while they are not constrained near $u=1$ in (9). In the following discussion however, we shall consider Eq.(9) and (11) together with the other ones differing only by the quadratic term in the noise as belonging to the same class of equations. The restrictive condition will be that no unphysical fluctuations be generated in the range spanned by the mean-field solution, i.e. the overall noise factor should stay positive in that range, such as for Eqs.(1].9).

Once this condition satisfied, the fluctuations are in general expected to be small in the "dense system" and without strong effect while they have an important role at small $u$ or $w$ which is the region of the dilute system. We will focus on this region in our study, and thus conjecture that our result is valid for the whole abovementionned class of Langevin equations with different quadratic term in the noise contribution. We will come back to this point in the discussion of our results.

In the following we will use the diffusive approximation of the QCD equation. However, one should keep in mind that a complete treatment of the fluctuations beyond the coarse-grained and fixed coupling approximations is still an open problem, since higher-order QCD contributions may change the features of the solution 2 .

\footnotetext{
${ }^{2}$ The effect of introducing the running of the QCD coupling constant in the QCD evolution equations with noise has been recently studied either through a numerical modelization 22] or on the BK equation with noise 23]. It seems that the fluctuation effects are transferred to higher rapidities than with fixed coupling.
} 


\section{Branching-Annihilating Random Walks}

As already mentionned the existence of a critical activation parameter has been found in BARW [14] together with an estimate of its value within a $2-d$ expansion at first order. An improved value could be found with higher order corrections or within the exact renormalization group approach [24]. We postpone this evaluation to further work.

For sake of generality, let us start by considering a reaction-diffusion process in dimension $d$, including the transition processes $A \rightarrow A+A$, with rate $\lambda, A+A \rightarrow(0, A)$ with rates $\left(\mu, \mu^{\prime}\right)$ respectively, and $A_{i} \rightarrow A_{i \pm 1}$ (where $i \pm 1$ is a short-hand notation for nearest neighbours in dimension $d$ ) with diffusion rate $D$. One writes the following action [15] for the probability distribution $s$ of the species $A$

$$
S[s, \bar{s}]=\int d^{d} x d t\left\{\bar{s}\left(\partial_{t}-D \nabla^{2}\right) s-\lambda_{\mathcal{R}} \bar{s} s-\lambda \bar{s}^{2} s+\left(2 \mu+\mu^{\prime}\right) \bar{s} s^{2}+\left(\mu+\mu^{\prime}\right) \bar{s}^{2} s^{2}\right\}
$$

where $\lambda_{\mathcal{R}}$ takes into account the extra process $A \rightarrow 0$ which is anyway imposed by renormalization of the $A \rightarrow A+A$. A general study of the reaction-diffusion action (11) in terms of $4-d$ expansion [15] is possible but the critical dimension being $d_{c}=4$, (with the noticable exception of the BARW process) the study we perform in dimension $d=1$ is not trivial. We will discuss further this point in the last section.

Let us then consider more specifically the BARW transition processes, which has the interesting special feature [14 to possess a critical dimension $d_{c}=2$. The BARW processes are described by the action (11) restricted to $\mu^{\prime}=0$, i.e. no coagulation process $A+A \rightarrow A$. Using the same formalism [15, 16], and considering for the present being the bare action $\left(\lambda_{\mathcal{R}}=\lambda\right)$, the theory corresponds to the Langevin equation

$$
\frac{\partial s}{\partial t}=D \nabla^{2} s+\lambda s-2 \mu s^{2}+\sqrt{2 \lambda s-2 \mu s^{2}} \eta(x, t),
$$

This Langevin equation is well-defined i.e. the noise factor is real for $s \leq \lambda / \mu$, while the "active state", which is the maximum value of $s$ in the mean-field approximation, is twice as small as the value $\lambda / 2 \mu$ where the noise is again zero. The noise is non zero at the stable fixed point $s=1$ of the meanfield equation but only very exceptional fluctuations could cause positivity problems under the square root. As already mentionned, we expect this 
feature not to be relevant for our study. The Eq.(12) belongs to the same class of Langevin equations as (11) and (9) that we have considered, and thus the results obtained with the BARW process will be expected to be valid for the other cases. Through the redefinition

$$
s=\frac{\lambda}{2 \mu} w ; \quad \epsilon=\sqrt{4 \mu}
$$

we indeed recover the familiar form of these equations, namely the same as (1), up to the quadratic term in the noise being now $\epsilon \sqrt{w-\frac{w^{2}}{2}} \nu(x, t)$.

In Ref. [14, the fluctations are shown to renormalize the activation parameter $\lambda$ through the one-loop effect $A \rightarrow A+A \rightarrow 0$, implying a counter-term in the action (12) corresponding to the new transition $A \rightarrow 0$. As one may infer, the renormalization contributes with a negative sign, showing that the fluctuations tend to stabilize the classically unstable fixed point.

Let us now show that the minimum obtained for the activation term in Ref.[14] corresponds to a maximal noise and evaluate its value.

After field-theoretical renormalization [14] using the 2-d expansion at first order, one finds the renormalized value $\lambda_{R}$ of the activation parameter

$$
\lambda_{R}=\lambda\left\{\frac{1-\frac{\mu}{2 \pi(2-d)}(\lambda D)^{-1+d / 2}}{1+\frac{\mu}{2 \pi(2-d)}(\lambda D)^{-1+d / 2}}\right\} .
$$

This induces, when $\lambda_{R}=0$ and $d \leq 2$ a minimum value

$$
\lambda_{\min }=\frac{1}{D}\left(\frac{\mu}{2 \pi}\right)^{\frac{2}{2-d}}
$$

below which there is no transition from the inactive to the active state. In terms of the directed percolation phase transition which is met at this point, the system no more percolates.

From the point of view of the Langevin formalism of (12) in $d=1$ and its solutions, it means that for

$$
\lambda_{\min }=\frac{1}{D}\left(\frac{\mu}{2 \pi}\right)^{2}
$$

there is an extinction of the noisy traveling waves.

Considering now the dimensionless noise $\tilde{\epsilon} \equiv \epsilon /(D \lambda)^{1 / 4}$, as defined in [1], and the parametric relations (13), one finds:

$$
\tilde{\epsilon} \equiv \frac{\epsilon}{(D \lambda)^{1 / 4}} \leq \tilde{\epsilon}_{\max }=\left(\frac{16 \mu^{2}}{D \lambda_{\min }}\right)^{1 / 4}=\sqrt{8 \pi} .
$$


In fact, the key point of this maximal noise property is that when the noise is strong enough, the inactive phase $(s=0)$ from being originally unstable in the mean-field or with noise weaker than the maximal one becomes stable when the noise strength reaches the value (17). This prevents the instability which leads to the noisy traveling waves going towards the stable fixed point. This stabilisation by the noise is a phenomenon appearing under various forms in statistical physics (see e.g. [25]).

Note that the value (17) has been obtained from the evaluation [14] of the minimum activation parameter at first order in the $2-d$ expansion of the renormalization procedure. A more precise estimate could be probably obtained from higher-order calculations or from the exact renormalization group analysis [24]. The best hope would be an exact solution in $d=1$.

\section{Applications to sFKPP and QCD}

\subsection{Maximal noise for sFKPP traveling waves}

In Ref.[11], the sFKPP equation is studied by using duality properties with the $A \leftrightarrow A+A$ reaction-diffusion process with creation or recombinaison, each with its own rate. At strong coupling, this dual reaction-diffusion process goes to a limiting coalescence regime, where at most one walker occupies a lattice site and with a probability of giving birth to another one in a neighbouring site. This limit allows one to find exact analytic solutions [10]. The resulting average wave speed, in terms of $v_{c}=2 \sqrt{D \lambda}$ the speed in the absence of noise [5, 16] and $\tilde{\epsilon}=\epsilon /(D \lambda)^{\frac{1}{4}}$ the dimensionless noise strength (17) takes the form [11].

$$
v=\frac{2 D \lambda}{\epsilon^{2}}=2 \sqrt{D \lambda} \times \frac{\sqrt{D \lambda}}{\epsilon^{2}}=\frac{v_{c}}{\tilde{\epsilon}^{2}} .
$$

The comparison with numerical simulations has been done in Ref.[11] and reproduced in Fig.2. It shows the following features: in the range of weak noise, the normalized speed agrees quite well with the theoretical predictions [5]. With larger noise strength, one can observe that, when $\mathcal{O}(1) \leq \tilde{\epsilon} \leq$ $\mathcal{O}(5-10)$, the numerically measured speed agrees reasonably well, within some statistical spread, with the theoretical prediction using the coalescence approximation.

However the end of the spectrum, not discussed in Ref.[11, seems to occur, compatible with a maximal noise limit. Indeed, the average speed of 
the numerical solution appears to stop near, indeed slightly superior, to the $2-d$ expansion value $\sqrt{8 \pi} \sim 5$, see Fig. 2 . We thus note that the approximate value of $\tilde{\epsilon}_{\max }$ is within the range of our estimate from the BARW process. It is an interesting challenge bpth from the numerical and theoretical point of view, to check whether more refined evaluations could confirm this finding. We hope to improve the this value by going beyond the 1-loop result or via the exact renormalization-group approach [24].

This region of noise strength below but near the transition where fluctuations stabilize the unstable mean-field fixed point corresponds to the critical region where the diffusion-reaction system meets the critical line of a phase transition of the directed percolation class [14, 24]. Hence, beyond the determination of the end-point of the noise strength, it would be interesting to discuss the other predictions coming from the renormalization properties at the phase transition [15], for instance the relevance of the critical exponents to the description of the sFKPP solution and the matching with the nearby coalescence limit approximation of the related reaction-diffusion process. This study is certainly deserved for the future.

\subsection{Maximal noise for QCD traveling waves}

Coming back to the problem of QCD traveling waves with strong fluctuations, we have already noticed (see (8) ) that in the diffusive approximation of the QCD equation there is a change of space variable

$$
x=L-A_{1} \alpha_{s} Y,
$$

where $A_{1}$ is the (negative) coefficient of the kernel expansion (7).

Hence, in the absence of noise, Eq.(19) amounts to a shift for the wave speed

$$
v_{Q C D}^{\text {no noise }}=v_{c}\left(1-\frac{\left|A_{1}\right|}{2 \sqrt{A_{0} A_{2}}}\right),
$$

with respect to the critical value $v_{c}=2 \sqrt{A_{0} A_{2}}$.

In the noisy regime dual to coalescence one finds [12]

$$
\frac{v_{Q C D}^{\text {strong noise }}}{v_{c}}=\frac{1}{(\tilde{\epsilon})^{2}}-\frac{\left|A_{1}\right|}{2 \sqrt{A_{2} A_{0}}} \leq \frac{1}{\left(\tilde{\epsilon}_{\max }\right)^{2}}-\frac{\left|A_{1}\right|}{2 \sqrt{A_{2} A_{0}}},
$$

where we have given the limit corresponding to the maximal sFKPP noise. Following (21) and the expression (17) of the maximal dimensionless noise 
strength, the normalized speed of the average QCD wave depends critically on the value of the ratio $\rho=\frac{\left|A_{1}\right|}{2 \sqrt{A_{0} A_{2}}}$ compared to the maximal noise strength $\tilde{\epsilon}_{\max }$.

- If $\tilde{\epsilon}_{\max }^{-2} \leq \rho \leq 1$ : The QCD speed goes to zero for a value of the noise strength which has not reached the value corresponding to (17); The system does not reach the phase transition for positive speed (required by the physical interpretation of a rapidity evolving gluon distribution.

- $\rho \leq \tilde{\epsilon}_{\max }^{-2}$ : The system reaches the critical point where appears the directed percolation phase transition where the wave traveling stops. The behaviour of the solution near the endpoint should be given by the renormalization group analysis.

In fact, if one sticks to the QCD leading-log order BFKL kernel (15) and the value $\tilde{\epsilon}_{\max } \approx 5-10$ extracted from the simulations [11], one sees that we are in the case of the first item: the directed percolation transition point cannot be reached. Indeed, in Ref.[12], two options were discussed for the diffusive approximation of the kernel for the strong-noise region, depending on which value of $\gamma_{c}$ the kernel has to be developped up to the second derivative. If one considers the same critical value $\gamma_{c}$ as in the no noise case, one finds with $\left(A_{0}, A_{1}, A_{2}\right)=(9.55,-25.6,24.3)$ and thus $\rho \sim .9$. If one considers a small value of $\gamma_{c} \ll 1$, one finds $\left(A_{0}, A_{1}, A_{2}\right) \sim\left(3 \gamma_{c}^{-1},-3 \gamma_{c}^{-2}, 1 \gamma_{c}^{-3}\right)$ and thus $\rho=\sqrt{3} / 2$, independently of $\gamma_{c}$. In both cases we find $\rho \approx .8 \sim .9<$ $\tilde{\epsilon}_{\text {max }} \approx 5-10$, and thus, referring to Fig 2 , the system of QCD traveling waves ends (reaching $v_{Q C D}=0$ ) within the coalescence regime, without reaching the sFKPP maximal nois屯 3 .

In order to give some appropriate estimate, using relation (10), and writing $\epsilon<\epsilon_{\max }^{Q C D}$, one finds the relation

$$
\frac{2 \alpha_{s}}{\pi} \sqrt{\kappa}<\left(\frac{2 A_{2}}{A_{0}}\right)^{\frac{1}{2}} .
$$

Hence, for $\frac{A_{0}}{A_{2}} \sim \mathcal{O}(1)$ the maximal noise could be reached even in the perturbative regime $\left(\frac{2 \alpha_{s}}{\pi}\right.$ small $)$ if the fudge factor $\kappa$ is large enough. On the other hand, it is interesting to consider the effect of increasing the coupling

\footnotetext{
${ }^{3}$ The result could change with corrections from higher QCD pertubative orders but would need a profound modification of the kernel. One could also consider yet unknown strong $\alpha_{s}$ coupling effects.
} 
constant $\alpha_{s}$. We see that in our framework it leads to an increase of the noise and consequently a decrease of the wave speed. Such an effect is otherwise expected in QCD when considering the transition towards the non-perturbatice regime. Both considerations give an incentive to developping further a coalescence model starting from the original QCD formukation e.g. directly in terms of branching annihilation and merging of gluons or QCD dipoles.

\section{Summary of results and discussion}

In the present note, which used some known results on the field-theoretical treatment of stochastic Langevin equations of sFKPP type and of related reaction-diffusion systems, we have found the existence of a limiting maximum strength of fluctuations, which can be quantified by a maximum value of the noise parameter in the Langevin equations. Below this value, there exists noisy traveling wave solutions which describe the transition of the system from an "inactive" phase to an "active" phase. Beyond this point, the fluctuations are strong enough to prevent the system to undergo such a transition and it stays in the inactive phase, which is otherwise unstable. Hence, we have yet another striking example of random fluctuations contributing to stabilize a deterministic unstable point.

Pursuing the analogies with the associated reaction-diffusion systems, the critical value of the noise corresponds to a directed percolation critical point, where the systems ceased to percolate. In this framework the noisy traveling waves could be interpreted as an average over the percolation path histories.

We have discussed two applications of the results. In the sFKPP case, we interpreted the existing numerical simulations showing the compatibility with the existence of a maximal noise strength in the range predicted by the fieldtheoretical calculations. For traveling waves in the diffusive approximation of the QCD evolution equations, the traveling waves go to zero before the transition point of the sFKPP analoguous equation due to a shift of the speed (at least for the leading order kernel in $\alpha_{s}$ ). The maximal noise-strength occurs in the region related by duality with a coalescence, which is accessible to exact noisy traveling wave solutions, as discussed in [12]. For large enough QCD fudge factor, it may be reached even in the perturbative QCD regime. Otherwise, it may indicate the slowing down of rapidity evolution expected from the nonperturbative QCD regime.

An important aspect of the discussion is the extent to which our result on 
the maximal noise is "universal", that is valid for a whole class of processes.

In our study we have met different Langevin equations, namely Eq.(11) corresponding to sFKPP, Eq.(9) corresponding to the coarse-graining of the QCD evolution equation in the diffusive approximation and finally Eq.(12) corresponding to the BARW process and for which we could extract the value of the maximal noise. Since these Langevin equations are all well defined and differ only by a quadratic term in the noise which is not relevant for the region near the unstable mean-field fixed point, we have conjectured that the result on the maximal noise should be the same, and thus given by (17) in the first-order approximation of the $2-d$ expansion for the BARW process.

Since each of these Langevin equations corresponds to the continuum limit of a specific reaction-diffusion process with different parameters for merging, splitting and annihilation, it is interesting to address directly the universality property in terms of a renormalization problem of the field theories for these three cases. By extension, one may investigate the validity of the obtained maximal noise strength for all reaction-diffusion processes pertaining to the universality class of the directed percolation processes [15], for the class of processes for which the formulation in terms of a Langevin equation differs only by a quadratic term in the noise (unless leading to imaginary noise term), which is irrelevant near the directed percolation transition point.

This task is not easy in the framework of the $d_{c}-d$ expansion since the critical dimension is $d_{c}=4$ in the directed percolation class, which is the universality class of the phase transition of these reaction-diffusion processes. It is a long way towards $d=1$. However, a qualitative general argument can be given which relies on the topological property of random walks in dimension $d<2$. Indeed, in that condition, any two random walks will have probability 1 to meet again. Hence, if the combination of reaction-diffusion constants contribute to a large enough dimensionless noise strength, one expects a stabilization of the otherwise unstable fixed-point. Starting with the general expression (11), and following a similar discussion as for the BARW case, one is led to conjecture $\tilde{\epsilon}_{\max }=\sqrt{8 \pi}+$ (higher order contributions). It is thus deserving to check this result in $d=14$.

\footnotetext{
${ }^{4}$ Note added in proof; The special case $d=0$ has been reconsidered very recently. In Ref. [26], the authors examine the diffusion-reaction systems with various couplings in the zero transverse dimension approximation. They find that only the reversible process $A \rightarrow A+A, A+A \rightarrow A$ leads to an increase of the solution with time (or the amplitude with rapidity in QCD language). They conclude that only the sFKPP equation (in its reduction to zero transverse dimension) to which this equation is related gives a satisfactory
} 
As an outlook one could propose two distinct directions for future work. In the sFKPP case, it would be useful to develop the connection between the regime just below the maximal noise with the results obtained by the renormalization group method near the directed percolation critical point. In particular the knowledge of the critical exponents should give interesting predictions for the behaviour of the averaged traveling wave and its eventual deformation at the critical point. From the QCD point of view, the existence of a maximal noise-strength at least 10 time smaller than the sFKPP one, leads to interesting predictions from the coalescence process via duality properties [10, 11]. It would be interesting to develop on the already known studies 12 by looking to dual properties similar to the coalescence limit more directly from the fromulation of QCD evolution processes in terms of merging, splitting and annihilation of gluons or QCD dipoles.

\section{Acknowledgements}

We thank Peter Smereka for allowing us to reproduce the Fig.2 containing the numerical results of Ref.[11]. We are indepted to Guillaume Beuf, Cyrille Marquet, Kirone Mallick and Gregory Soyez for careful reading of the manuscript, many useful suggestions and for constructive criticisms. Discussions with Léonie Canet and Bertrand Delamotte are acknowledged. We thank José Guilherme Milhano for communicating the Ref.[26] prior to publication.

\section{References}

[1] R. A. Fisher, Ann. Eugenics 7, 355 (1937); A. Kolmogorov, I. Petrovsky, and N. Piscounov, Moscou Univ. Bull. Math. A1, 1 (1937).

toy model of high-energy QCD. We note that in our case $d=1$, the diffusion term (absent in zero transverse dimension) is crucial for the properties of the process, especially in the dilute region. For instance the reduced noise $\tilde{\epsilon}$ becomes infinite at zero diffusion constant. The properties reported in [26] seeem to be related with the noise in the region of the other mean-field fixed point (and thus depending critically on the quadratic term in the noise expression, contrary to the $d=1$ case), which is leading to negative evolution in time, except for the reduction of the sFKPP equation in $d=0$. 
[2] S. Munier, Nucl. Phys. A 755 (2005) 622 arXiv:hep-ph/0501149]. E. Iancu, A. H. Mueller and S. Munier, Phys. Lett. B 606 (2005) 342 arXiv:hep-ph/0410018.

[3] M. Bramson, Mem. Am. Math. Soc. 44, 285 (1983).

[4] U. Ebert, W. van Saarloos, Physica D 146, 1 (2000).

[5] E. Brunet and B. Derrida, Phys. Rev. E56, 2597 (1997).

[6] G. Soyez, Phys. Rev. D 72, 016007 (2005) arXiv:hep-ph/0504129.

[7] E. Brunet, B. Derrida, A. H. Mueller and S. Munier, Phys. Rev. E 73, 056126 (2006) arXiv:cond-mat/0512021.

[8] A. M. Staśto, K. Golec-Biernat, and J. Kwiecinski, Phys. Rev. Lett. 86, 596 (2001), hep-ph/0007192.

[9] Y. Hatta, E. Iancu, C. Marquet, G. Soyez and D. N. Triantafyllopoulos, Nucl. Phys. A 773, 95 (2006) arXiv:hep-ph/0601150, E. Iancu, C. Marquet and G. Soyez, Nucl. Phys. A 780, 52 (2006) arXiv:hep-ph/0605174.

[10] D. ben-Avraham, M. A. Burschka and C. R. Doering, J. Stat. Phys. 60,695 (1990); C. R. Doering, M. A. Burschka and W. Horsthemke, J. Stat. Phys. 65,953 (1991).

[11] C. R. Doering, C, Mueller and P. Smereka, Phys. A 325, 325 (2003).

[12] C. Marquet, R. Peschanski and G. Soyez, Phys. Rev. D 73, 114005 (2006) arXiv:hep-ph/0512186.

[13] M. Doi, J. Phys. A 9, 1479 (1976) L. Peliti, J. Phys. (Paris) 46, 1469 (1985)

[14] J. L. Cardy, U. C. Täuber, J. Stat. Phys. 90,1 (1998); ArXiv:cond-mat/9704160.

[15] U. C. Tauber, M. Howard and B. P. Vollmayr-Lee Phys. A 38: R79 (2005); ArXiv:cond-mat/0501678.

[16] L. Pechenik and H. Levine Phys. Rev. E 59, 3893 (1999). 
[17] E. Iancu and R. Venugopalan, "The color glass condensate and high energy scattering in QCD," arXiv:hep-ph/0303204.

[18] E. Iancu and D. N. Triantafyllopoulos, Nucl. Phys. A 756, 419 (2005) arXiv:hep-ph/0411405].

[19] L. N. Lipatov, Sov. J. Nucl. Phys. 23, 338 (1976); E. A. Kuraev, L. N. Lipatov, and V. S. Fadin, Sov. Phys. JETP 45, 199 (1977); I. I. Balitsky and L. N. Lipatov, Sov. J. Nucl. Phys. 28, 822 (1978).

[20] I. I. Balitsky, Nucl. Phys. B 463, 99 (1996) arXiv:hep-ph/9509348]; Y. V. Kovchegov, Phys. Rev. D60, 034008 (1999), hep-ph/9901281;

[21] S. Munier and R. Peschanski, Phys. Rev. Lett. 91, 232001 (2003) arXiv:hep-ph/0309177]; Phys. Rev. D69, 034008 (2004) arXiv:hep-ph/0310357|; Phys. Rev. D70, 077503 (2004) arXiv:hep-ph/0310357].

[22] A. Dumitru, E. Iancu, L. Portugal, G. Soyez and D. N. Triantafyllopoulos, JHEP 0708, 062 (2007) arXiv:0706.2540 [hep-ph]].

[23] G. Beuf, "Asymptotics of QCD traveling waves with fluctuations and running coupling effects," arXiv:0708.3659 [hep-ph].

[24] L. Canet, B. Delamotte, O. Deloubrière, N. Wschebor, Phys.Rev.Lett. 92 (2004) 195703; L. Canet, H. Chaté, B. Delamotte, Phys.Rev.Lett. 92 (2004) 255703; L. Canet, H. Chaté, B. Delamotte, I. Dornic, M. A. Munõz, Phys. Rev. Lett. 95, 100601 (2005).) B. Delamotte, L. Canet Condensed Matter Phys. 8 (2005) 163; L. Canet, H. Chaté, arXiv:cond-mat/0610468]; L. Canet, J. Phys. A: Math. Gen.39, 7901 (2006); For a comprehensive review, see e.g. B. Delamotte, "An Introduction to the Nonperturbative Renormalization Group", ArXiv:cond-mat/0511456.

[25] K. Mallick and P. Marcq, Eur. Phys. J. B. 36, 119 (2003), 38, 99 (2004).

[26] N. Armesto, S. Bondarenko, J. G. Milhano and P. Quiroga, "Reactiondiffusion processes in zero transverse dimensions as toy models for highenergy QCD," arXiv:0803.0820 [hep-ph]. 


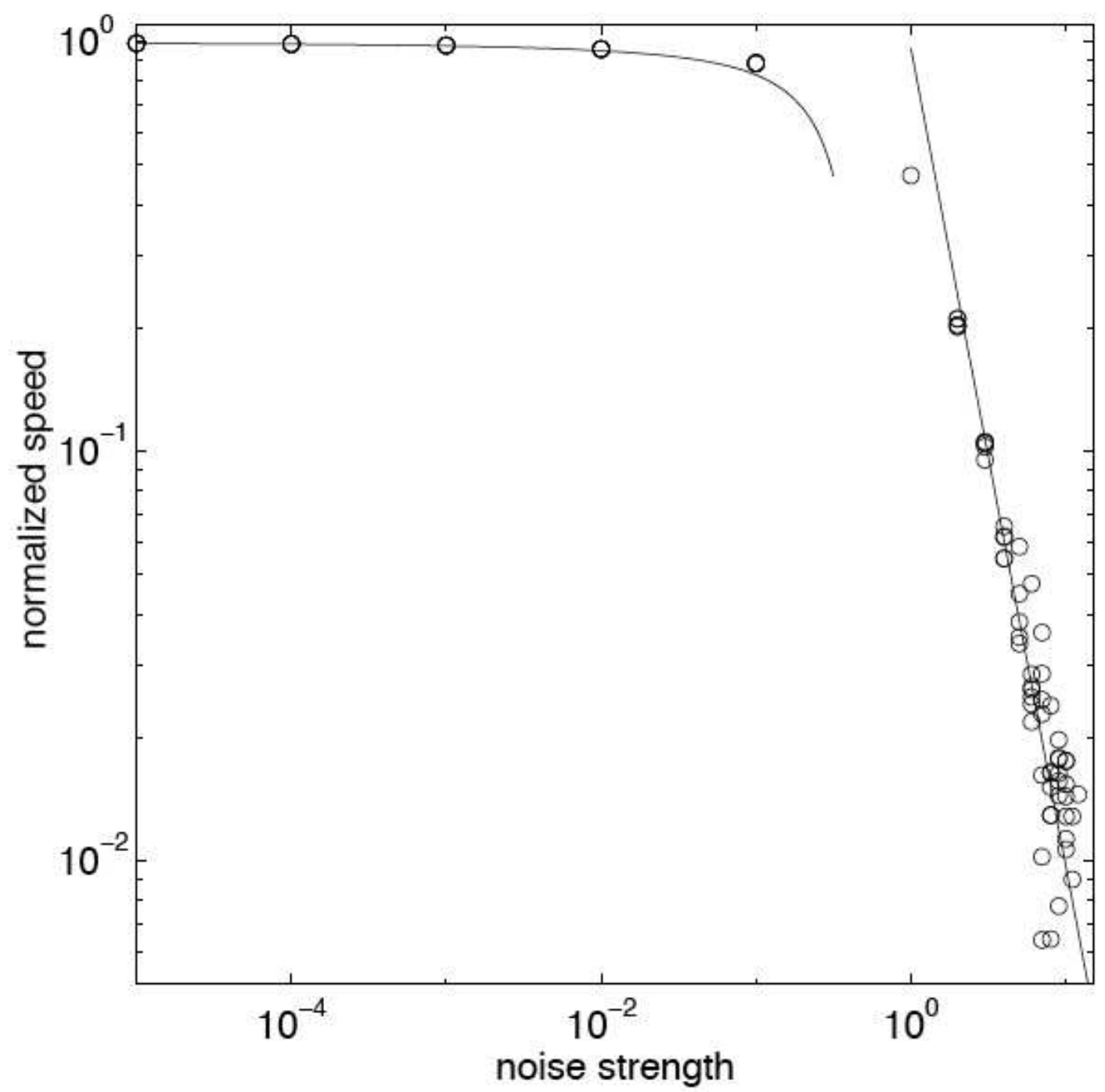

Figure 2: Wave speed as a function of the noise strength for the solutions of the sFKPP equation, from ref.[11].

Vertical axis: traveling wave speed normalized to the critical one $2 \sqrt{D \lambda}$ of the deterministic FKPP equation. Horizontal axis: dimensionless noise strength $\bar{\epsilon}=\epsilon /(D \lambda)^{\frac{1}{4}}$. Line on the left: the weak noise prediction [5, 16]; Line on the right: the strong noise prediction [10]. One observes a maximal speed around a noise strength of order 10 . 